\title{
Comparison between squamous cell carcinoma and inflammatory diseases of the oral and maxillofacial region using gallium- 67 scintigraphy with computed tomography and magnetic resonance imaging
}

\author{
Ichiro Ogura ${ }^{1,2 A, E}$, Takaaki Oda ${ }^{1 B, F}$, Mikiko Sue ${ }^{1 B, F}$, Yoshihiko Sasaki ${ }^{1 C, D}$, Kazuhide Hayama ${ }^{2 C, D}$ \\ ${ }^{1}$ Radiology, The Nippon Dental University Niigata Hospital, Niigata, Japan \\ ${ }^{2}$ Department of Oral and Maxillofacial Radiology, The Nippon Dental University School of Life Dentistry at Niigata, Niigata, Japan
}

\begin{abstract}
Purpose: This study aimed to compare squamous cell carcinoma (SCC) with inflammatory diseases of the oral and maxillofacial region using gallium $67\left({ }^{67} \mathrm{Ga}\right)$ scintigraphy with computed tomography $(\mathrm{CT})$ and magnetic resonance imaging (MRI).

Material and methods: Seventy patients with SCC and 15 patients with inflammatory diseases of the oral and maxillofacial region underwent ${ }^{67} \mathrm{Ga}$ scintigraphy with CT and MRI. A comparison between imaging features of ${ }^{67} \mathrm{Ga}$ scintigraphy, CT, and MRI and lesions was performed with the Pearson's chi-squared test.

Results: ${ }^{67} \mathrm{Ga}$ scintigraphy was positive for 25 of 39 patients with SCC positive on CT and/or MRI (64.1\%) and for 13 of 15 patients with inflammatory diseases positive on CT and/or MRI (86.7\%). The detection of inflammatory diseases with ${ }^{67} \mathrm{Ga}$ scintigraphy was higher than that of SCC $(p=0.104)$.

Conclusions: This study compared SCC with inflammatory diseases of the oral and maxillofacial region using ${ }^{67} \mathrm{Ga}$ scintigraphy with CT and MRI. ${ }^{67} \mathrm{Ga}$ scintigraphy is an effective technique for detection of inflammatory diseases of the oral and maxillofacial region.
\end{abstract}

Key words: gallium radioisotopes, gamma cameras, carcinoma, inflammation.

\section{Introduction}

Gallium $67\left({ }^{67} \mathrm{Ga}\right)$ scintigraphy is a useful adjunct tool for differentiation of malignant tumours from benign tumours or inflammatory disease in the oral and maxillofacial region [1]. ${ }^{67} \mathrm{Ga}$ scintigraphy is an effective technique for the evaluation of head and neck squamous cell carcinoma (SCC), especially tumour recurrence and distant metastases [2]. Furthermore, ${ }^{67} \mathrm{Ga}$ single-photon emission tomography (SPECT) substantially increases confidence in the diagnosis of head and neck tumours when comput- ed tomography (CT) and/or magnetic resonance imaging (MRI) do not permit differentiation between benign and malignant disease [3]. However, in recent years, positron emission tomography (PET) using the radiolabelled glucose analogue ${ }^{18} \mathrm{~F}$-fluorodeoxyglucose (FDG) has shown potential to detect distant metastases [4-7].

Apart from SCC, some authors have reported that ${ }^{67} \mathrm{Ga}$ scintigraphy is useful in the differentiation of malignant lymphoma [8], sarcoidosis [9-11], and other inflammatory diseases $[12,13]$. However, to the best of our knowledge, ${ }^{67} \mathrm{Ga}$ scintigraphy with multimodal imaging, such as

Correspondence address:

Ichiro Ogura, Radiology, The Nippon Dental University Niigata Hospital, 1-8 Hamaura-cho, Chuo-ku, Niigata, Niigata 951-8580, Japan,

phone +81-25-267-1500, fax +81-25-267-1134, e-mail: ogura@ngt.ndu.ac.jp

Authors' contribution:

A Study design - B Data collection · C Statistical analysis · D Data interpretation - E Manuscript preparation · F Literature search · G Funds collection 
CT and MRI, for the comparison of SCC and inflammatory diseases of the oral and maxillofacial region has not been reported in the literature. The aim of this study was to compare SCC with inflammatory diseases of the oral and maxillofacial region using ${ }^{67} \mathrm{Ga}$ scintigraphy with $\mathrm{CT}$ and MRI.

\section{Material and methods}

\section{Patients}

The Ethics Committee of our institution approved this retrospective study. After providing written, informed consent, 85 patients ( 44 male, 41 female; range age 25-95 years, mean age 67.0 years; 70 patients with SCC, 15 patients with inflammatory diseases) underwent ${ }^{67} \mathrm{Ga}$ scintigraphy with CT and MRI at our university hospital from July 2013 to February 2018. The histopathological diagnoses were obtained by surgery or biopsy in all cases.

\section{Image acquisition}

CT imaging was performed with a 16-multidetector CT scanner (Aquilion TSX-101A; Canon Medical Systems, Otawara, Japan) using the oral and maxillofacial protocol at our hospital: tube voltage, $120 \mathrm{kV}$; tube current, 150 $\mathrm{mAs}$; field of view, $240 \times 240 \mathrm{~mm}$; rotation time, $0.50 \mathrm{sec}$. The protocol consisted of axial acquisition $(0.50 \mathrm{~mm})$ with axial, coronal, and sagittal multiplanar reformation (MPR) images. The patients received contrast-enhanced CT (CECT) with non-ionic iodine for head and neck lesions. One non-ionic contrast media was used: Iohexol 300 mgI/ml (Omunipaque 300 Syringe, Daiichi-Sankyo, Tokyo, Japan). Contrast medium was administered as an injection of $100 \mathrm{ml}$ at a rate of $2.0 \mathrm{ml} / \mathrm{s}$ (Autoenhance A-250, Nemoto-Kyorindo, Tokyo, Japan).

The MR images (1.5 Tesla MR unit; EXCELART Vantage MRT-2003; Toshiba Medical Systems, Otawara, Japan) with a head coil included unenhanced axial T1-[repetition time (TR) $660 \mathrm{~ms}$, echo time (TE) $12 \mathrm{~ms}$ ], T2-weighted imaging (TR $4000 \mathrm{~ms}$, TE $120 \mathrm{~ms}$ ), short TI inversion recovery images (TR $2500 \mathrm{~ms}$, TE $15 \mathrm{~ms}$, TI $190 \mathrm{~ms}$ ), as well as coronal T1-weighted and T2-weighted imaging. After an injection of contrast medium (gadobutrol; Gadovist $1.0 \mathrm{~mol} / \mathrm{l}$ Syringe, Bayer, Osaka, Japan; $0.1 \mathrm{ml} / \mathrm{kg}$ ), axial and coronal T1-weighted images (TR $660 \mathrm{~ms}$, TE $12 \mathrm{~ms}$ ) were acquired.

${ }^{67} \mathrm{Ga}$ scintigraphy was obtained with an SNC-5100R (Shimadzu, Kyoto, Japan) and a Scintipack 24000 (Shimadzu) with a $512 \times 512$ matrix at 72 hours after the injection; images were recorded on the computer at $6 \mathrm{~min} /$ frame. The radiopharmaceutical used in this study was ${ }^{67} \mathrm{Ga}$-citrate (Gallium Citrate-Ga67 Injection, FUJIFILM RI Pharma, Tokyo, Japan). Each patient was administered the agent at $185 \mathrm{MBq}$ with a rapid intravenous injection. The stored data were displayed on a screen for analysis.

\section{Image analysis}

For patients with SCC and inflammatory diseases of the oral and maxillofacial region, imaging features of ${ }^{67} \mathrm{Ga}$ scintigraphy, CT, and MRI were independently analysed by two oral and maxillofacial radiologists. Regarding ${ }^{67} \mathrm{Ga}$ scintigraphy, images of the lesions were classified into two groups: positive, where the intensity of ${ }^{67} \mathrm{Ga}$ in the lesion area was higher than that in the surrounding normal area, and negative, where the intensity of ${ }^{67} \mathrm{Ga}$ in the lesion area was the same as in the surrounding normal area. Any discrepancies of the imaging evaluation were resolved by consensus of the two oral and maxillofacial radiologists.

\section{Statistical analysis}

The comparison between imaging features of ${ }^{67} \mathrm{Ga}$ scintigraphy, CT, and MRI and lesions was performed with the Pearson's chi-squared test. These analyses were performed with the statistical package IBM SPSS Statistics, version 24 (IBM Japan, Tokyo, Japan). A $P$ value lower than 0.05 was considered statistically significant.

\section{Results}

Regarding cellulitis of the submandibular region (Figure 1), ${ }^{67} \mathrm{Ga}$ scintigraphy showed increased uptake. Axial contrast-enhanced CT (CECT) image showed a masslike lesion with heterogeneous enhancement. On MRI, axial T1-weighted image (T1WI) revealed homogeneous low-signal intensity. Post-contrast T1WI showed heterogeneous enhancement. Short TI inversion recovery (STIR) revealed heterogeneous high-signal intensity. Regarding neck metastases of SCC (Figures 2, 3), ${ }^{67} \mathrm{Ga}$ scintigraphy showed increased uptake. Axial CECT image showed rim enhancement. On MRI, axial T1WI revealed homogeneous low-signal intensity. Post-contrast T1WI showed rim enhancement. STIR revealed heterogeneous high-signal intensity. Furthermore, regarding sialadenitis of the submandibular gland (Figure 2) and parotid gland (Figure 3), ${ }^{67} \mathrm{Ga}$ scintigraphy showed increased uptake. Axial CECT image showed enhancement. On MRI, axial T1WI revealed homogeneous low-signal intensity. Post-contrast T1WI showed enhancement. STIR revealed heterogeneous high-signal intensity.

Table 1 shows the comparison between SCC and inflammatory diseases of the oral and maxillofacial region using ${ }^{67} \mathrm{Ga}$ scintigraphy with CT and MRI. ${ }^{67} \mathrm{Ga}$ scintigraphy was positive for 25 of 39 patients with SCC positive on CT and/or MRI (64.1\%), and for 13 of 15 patients with inflammatory diseases positive on CT and/or MRI (86.7\%). The detection of inflammatory diseases with ${ }^{67} \mathrm{Ga}$ scintigraphy was higher than that of SCC $(p=0.104)$.

\section{Discussion}

${ }^{67} \mathrm{Ga}$ scintigraphy has been widely used to detect various malignant neoplasms, such as SCC $[1,3]$ and malignant 

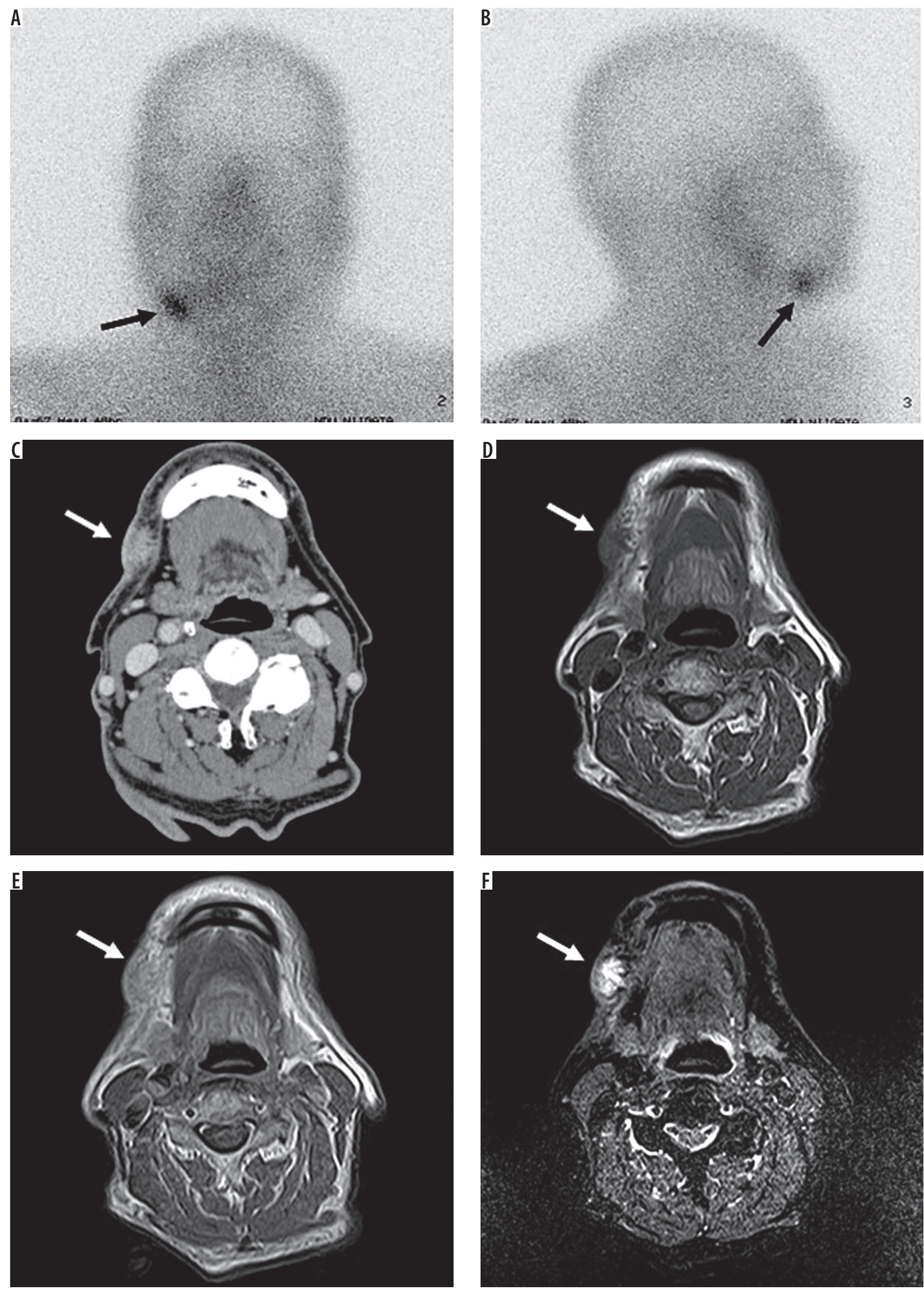

Figure 1. Cellulitis of the right side of the submandibular region in a 78-year-old male. ${ }^{67} \mathrm{Ga}$ scintigraphy shows increased uptake in the submandibular region (arrow) (A, B). Axial contrast-enhanced (T image shows a mass-like lesion with heterogeneous enhancement in the submandibular region (arrow) (C). On MRI, axial T1-weighted image (T1WI) revealed homogeneous low-signal intensity (arrow) (D). Post-contrast T1WI showed heterogeneous enhancement (arrow) (E). Short Tl inversion recovery (STIR) revealed heterogeneous high-signal intensity in the submandibular region (arrow) (F) 

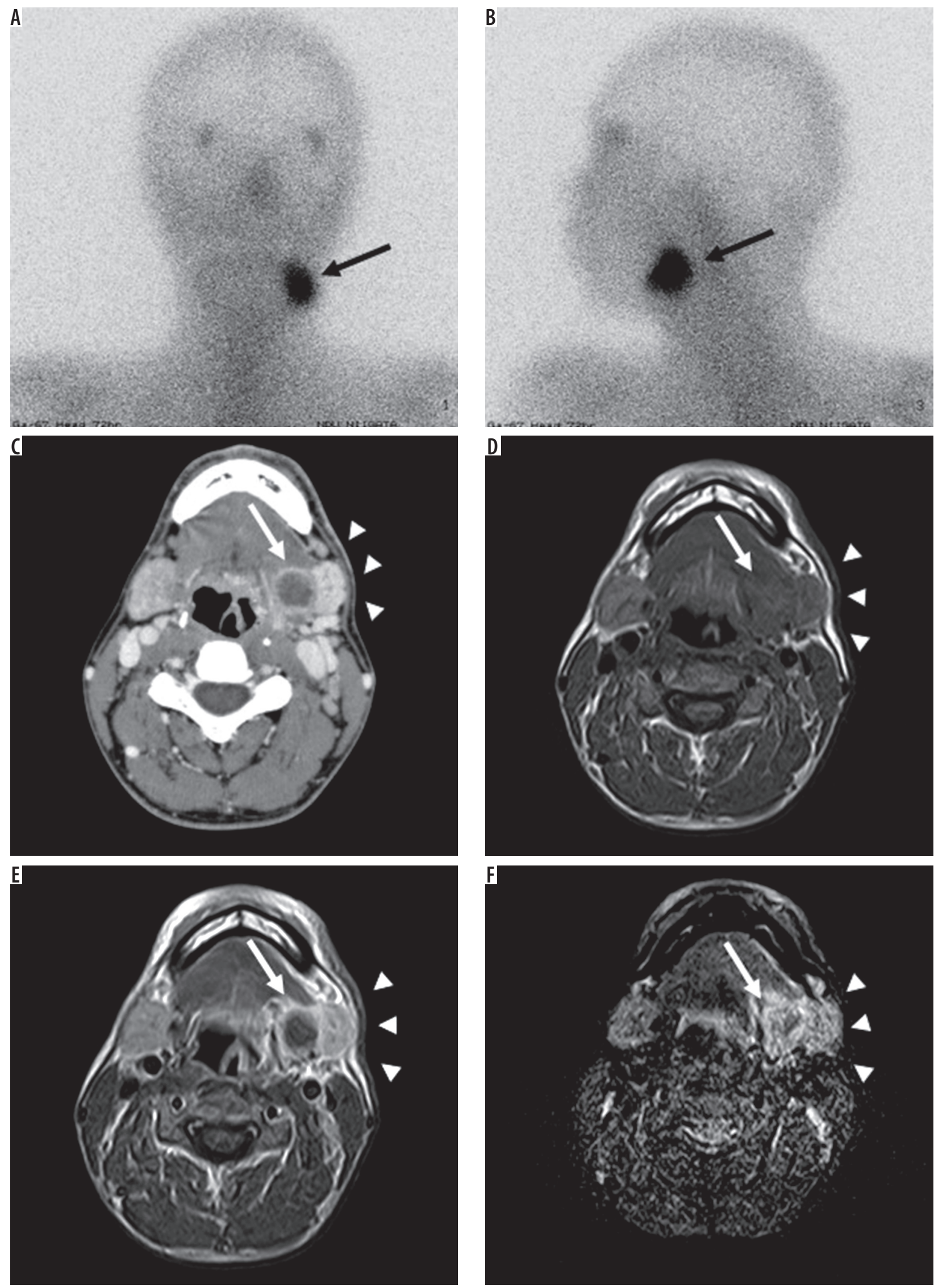

Figure 2. Squamous cell carcinoma of the left side of the tongue in a 39 -year-old male. ${ }^{67} \mathrm{G}$ a scintigraphy shows increased uptake in the submandibular region (arrow) (A, B). Axial contrast-enhanced CT image shows a submandibular lymph node with rim enhancement (arrow) and submandibular gland with enhancement (arrowheads) (C). On MRI, axial T1WI revealed a submandibular lymph node (arrow) and submandibular gland (arrowheads) with homogeneous low-signal intensity (D). Post-contrast T1WI showed a submandibular lymph node with rim enhancement (arrow) and submandibular gland with enhancement (arrowheads) (E). STIR revealed a submandibular lymph node (arrow) and submandibular gland (arrowheads) with heterogeneous high-signal intensity (F) 

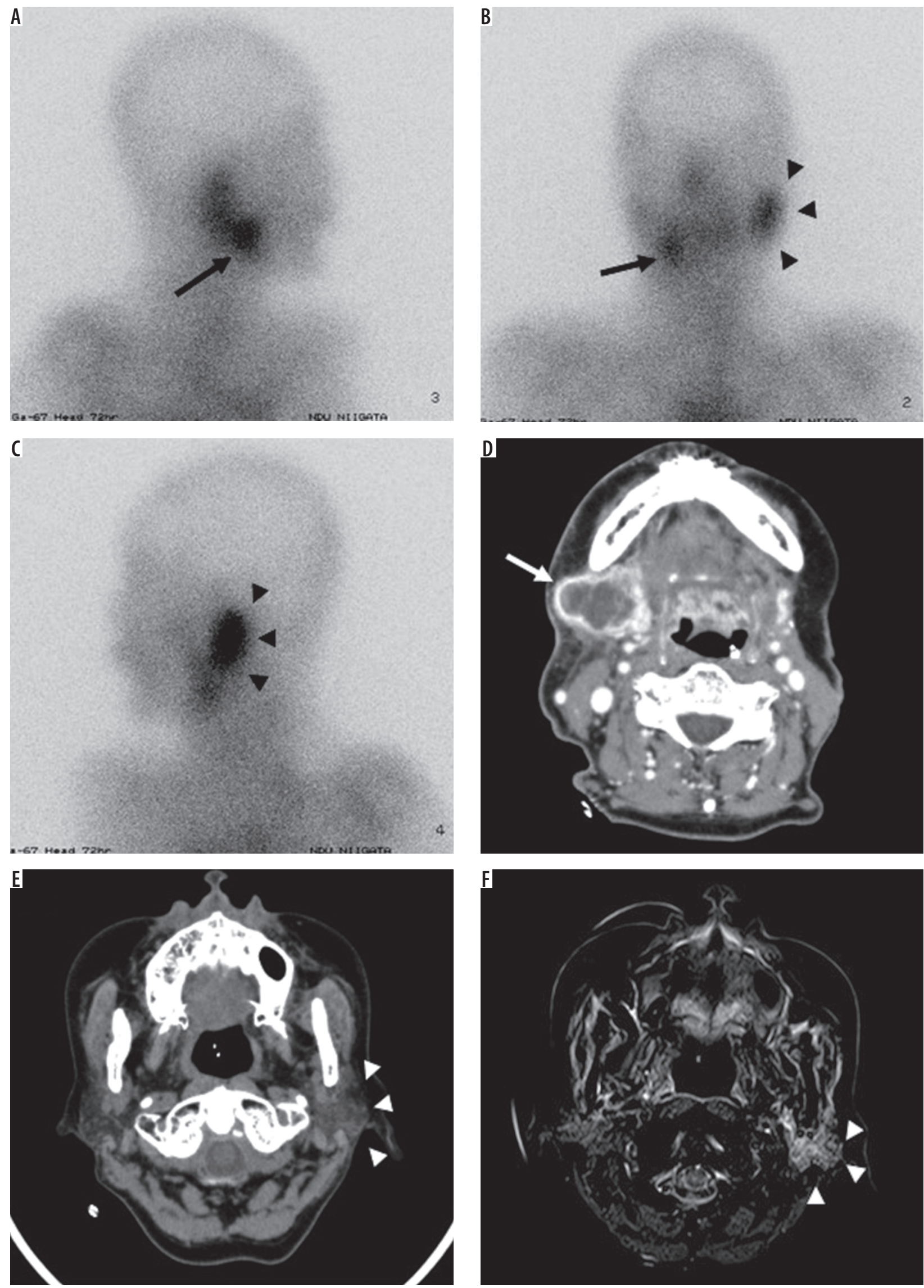

Figure 3. Squamous cell carcinoma of the right side of the tongue in a 70 -year-old female. ${ }^{67} \mathrm{Ga}$ scintigraphy shows increased uptake in the submandibular region (arrow) and buccal region (arrowheads) (A-C). Axial contrast-enhanced (T image shows a submandibular lymph node with rim enhancement (arrow) (D). Axial contrast-enhanced CT image shows a parotid gland with heterogeneous enhancement (arrowheads) (E). On MRI, axial STIR revealed parotid gland with heterogeneous high-signal intensity (arrowheads) (F) 
Table 1. Comparison between squamous cell carcinoma and inflammatory diseases of the oral and maxillofacial region using gallium-67 scintigraphy with CT and MRI

\begin{tabular}{|l|c|c|c|}
$\begin{array}{l}\text { Lesions positive } \\
\text { (number of cases) }\end{array}$ & $\begin{array}{c}\text { Scintigraphy negative } \\
\text { CT and/or MRI negative }\end{array}$ & $\begin{array}{c}\text { Scintigraphy negative } \\
\text { CT and/or MRl positive }\end{array}$ & $\begin{array}{c}\text { Scintigraphy } \\
\text { CT and/or MRI positive* }\end{array}$ \\
\hline \begin{tabular}{l} 
Inflammatory diseases (15) \\
\hline Osteoradionecrosis (7)
\end{tabular} & 0 & $2(13.3 \%)$ & $13(86.7 \%)$ \\
\hline $\begin{array}{l}\text { Cellulitis (4) } \\
\text { Medication-related osteonecrosis } \\
\text { of the jaw (MRONJ) (3) }\end{array}$ & 0 & $0(28.6 \%)$ & $5(71.4 \%)$ \\
\hline Actinomycosis (1) & 0 & $0(0 \%)$ & $3(100 \%)$ \\
\hline \begin{tabular}{l} 
Squamous cell carcinoma (70) \\
\hline Tongue (28)
\end{tabular} & 31 & $0(0 \%)$ & $1(100 \%)$ \\
\hline Buccal mucosa (8) & 18 & $14(35.9 \%)$ & $25(64.1 \%)$ \\
\hline Maxilla (8) & 5 & $8(80.0 \%)$ & $2(20.0 \%)$ \\
\hline Mandible (6) & 2 & $1(33.3 \%)$ & $2(66.7 \%)$ \\
\hline Floor of mouth (3) & 2 & $0(0 \%)$ & $6(100 \%)$ \\
\hline Lip (3) & 3 & $0(0 \%)$ & $4(100 \%)$ \\
\hline Neck metastases (13) & 1 & 0 & 0 \\
\hline Distant metastases (lung: 1$)$ & 0 & $1(50.0 \%)$ & $1(50.0 \%)$ \\
\hline
\end{tabular}

*Inflammatory diseases (86.7\%) vs. squamous cell carcinoma $(64.1 \%)(p=0.104)$

lymphoma [8] of the head and neck. In our study, ${ }^{67} \mathrm{Ga}$ scintigraphy was positive for 25 of 39 patients with SCC positive on CT and/or MRI (64.1\%). Regarding the mechanism of gallium-67 accumulation in tumours, Tsan et al. [14] showed that ${ }^{67} \mathrm{Ga}$ was delivered to the tumour through capillaries with increased permeability, and ${ }^{67} \mathrm{Ga}$ binding proteins might also contribute to the accumulation and retention of ${ }^{67} \mathrm{Ga}$ in tumours. In this study, ${ }^{67} \mathrm{Ga}$ scintigraphy was positive for two of 10 patients with tongue SCC positive on CT and/or MRI (20.0\%). We consider that the size of tumours is also a factor of the degree of ${ }^{67} \mathrm{Ga}$ accumulation in lesions. However, in recent years, ${ }^{18} \mathrm{~F}-\mathrm{FDG}$ PET/ $\mathrm{CT}$ is crucial for determining treatment strategy because it helps to avoid incorrect staging and also provides an accurate assessment of treatment response $[4,6]$.

In this study, ${ }^{67} \mathrm{Ga}$ scintigraphy was positive for 13 of 15 patients with inflammatory diseases positive on CT and/or MRI (86.7\%). Li et al. [1] indicated that ${ }^{67} \mathrm{Ga}$ scintigraphy was positive for two of 11 patients who had chronic inflammatory lesions (1/4 parotitis, 1/5 submaxillaritis and 0/2 lymphadenitis) (18.2\%). Tsan et al. [14] showed that some tumours may be taken up by inflammatory cells when they are present. Furthermore, Keijsers et al. [9] showed imaging of the inflammatory activity of sarcoidosis, namely overall sensitivity to detect active sarcoidosis, was $88 \%$ for ${ }^{67} \mathrm{Ga}$ imaging. Ishii et al. [10] showed that ${ }^{67} \mathrm{Ga}$ scintigraphy was useful in differentiating between sarcoidosis and IgG4-related disease. Tsai et al. [13] suggested that the kidney uptake index from the absolute quantitative renal ${ }^{67} \mathrm{Ga}$ scintigraphy may be a use- ful parameter for evaluating the disease activity in lupus nephritis. In this study, the detection of inflammatory diseases with ${ }^{67} \mathrm{Ga}$ scintigraphy was higher than that of SCC $(p=0.104)$. Consequently, the authors consider that ${ }^{67} \mathrm{Ga}$ scintigraphy was more useful for inflammatory diseases of the oral and maxillofacial region than SCC. Furthermore, we recommend ${ }^{67} \mathrm{Ga}$ scintigraphy with multimodal imaging, such as CT and MRI, for detection of SCC and inflammatory diseases of the oral and maxillofacial region.

There were several limitations of this study. The sample was relatively small. Therefore, further research is necessary to validate these results.

\section{Conclusions}

This study compared SCC with inflammatory diseases of the oral and maxillofacial region using ${ }^{67} \mathrm{Ga}$ scintigraphy with CT and MRI. ${ }^{67} \mathrm{Ga}$ scintigraphy is an effective technique for detection of inflammatory diseases of the oral and maxillofacial region.

\section{Acknowledgements}

The authors thank Professor Y. Okada, Department of Pathology, The Nippon Dental University School of Life Dentistry at Niigata and Professor A. Yamaguchi, Oral and Maxillofacial Surgery, The Nippon Dental University Niigata Hospital.

This work was supported by JSPS KAKENHI, Grant Number JP 18K09754. 


\section{Conflict of interest}

The authors report no conflict of interest.

\section{References}

1. Li N, Zhu W, Zuo S, et al. Value of gallium-67 scanning in differentiation of malignant tumors from benign tumors or inflammatory disease in the oral and maxillofacial region. Oral Surg Oral Med Oral Pathol Oral Radiol Endod 2003; 96: 361-367.

2. Murata Y, Ishida R, Umehara I, et al. 67 Ga whole-body scintigraphy in the evaluation of head and neck squamous cell carcinoma. Nucl Med Commun 1999; 20: 599-607.

3. Kosuda S, Kadota Y, Umeda S, et al. Does supplementation of CT and MRI with gallium-67 SPECT improve the differentiation between benign and malignant tumors of the head and neck? Ann Nucl Med 2003; 17: 475-480.

4. Kitagawa Y, Nishizawa S, Sano K, et al. Prospective comparison of 18F-FDG PET with conventional imaging modalities (MRI, CT, and 67 Ga scintigraphy) in assessment of combined intraarterial chemotherapy and radiotherapy for head and neck carcinoma. J Nucl Med 2003; 44: 198-206.

5. Corey AS, Hudgins PA. Radiographic imaging of human papillomavirus related carcinomas of the oropharynx. Head Neck Pathol 2012; 6: S25-40.

6. Plaxton NA, Brandon DC, Corey AS, et al. Characteristics and limitations of FDG PET/CT for imaging of squamous cell carcinoma of the head and neck: a comprehensive review of anatomy, metastatic pathways, and image findings. AJR Am J Roentgenol 2015; 205: W519-531.
7. Senft A, Hoekstra OS, Witte BI, et al. Screening for distant metastases in head and neck cancer patients using FDG-PET and chest CT: validation of an algorithm. Eur Arch Otorhinolaryngol 2016; 273: 2643-2650.

8. Okada M, Sato N, Ishii K, et al. FDG PET/CT versus CT, MR imaging, and $67 \mathrm{Ga}$ scintigraphy in the posttherapy evaluation of malignant lymphoma. Radiographics 2010; 30: 939-957.

9. Keijsers RG, Grutters JC, Thomeer M, et al. Imaging the inflammatory activity of sarcoidosis: sensitivity and inter observer agreement of $67 \mathrm{Ga}$ imaging and 18F-FDG PET. Q J Nucl Med Mol Imaging 2011; 55: 66-71.

10. Ishii S, Miyajima M, Sakuma K, et al. Comparison between sarcoidosis and IgG4-related disease by whole-body 67Ga scintigraphy. Nucl Med Commun 2013; 34: 13-18.

11. Shim H, Joo J, Choi HJ, et al. Lack of increased FDG uptake in the lacrimal and salivary glands in patients with sarcoidosis and potential underlying mechanism. Clin Nucl Med 2016; 41: 274-277.

12. Aslangul E, M'bemba J, Caillat-Vigneron N, et al. Diagnosing diabetic foot osteomyelitis in patients without signs of soft tissue infection by coupling hybrid $67 \mathrm{Ga}$ SPECT/CT with bedside percutaneous bone puncture. Diabetes Care 2013; 36: 2203-2210.

13. Tsai SC, Hsieh TY, Huang PW, et al. Absolute quantitative evaluation of 67Ga scintigraphy in lupus nephritis. Clin Nucl Med 2016; 41: 442-446.

14. Tsan MF, Scheffel U. Mechanism of gallium-67 accumulation in tumors. J Nucl Med 1986; 27: 1215-1219. 